\title{
On some theorems of Tarafdar
}

\section{S.A. Husain and V.M. Sehgal}

\begin{abstract}
In a recent paper (Bulz. Austral. Math. Soc. 13 (1975), 241-245), Tarafdar has considered nonexpansive self mappings on a subset $X$ of a locally convex vector space $E$ and proved an extension to $E$ of a theorem of Göhde. The purpose of this paper is to show that the condition $f: X \rightarrow X$, in Göhde-Tarafdar's Theorem in the above paper, may be weakened to $f: X \rightarrow E$ with $f(\partial X) \subseteq X$. As a consequence, it is further shown that an extension to $E$ of a well-known common fixed point theorem of Belluce and Kirk due to Tarafdar remains true on domains that are not necessarily bounded or quasi-complete.
\end{abstract}

\section{Introduction}

Let $X$ be a subset of a locally convex vector space $E$. In a recent paper [7], Tarafdar considered nonexpansive mappings $f: X \rightarrow X$ and proved extensions of certain results of Göhde [4], Taylor [8], and Belluce and Kirk [2]. The purpose of this paper is to show that the condition $f: X \rightarrow X$ in Göhde-Tarafdar's Theorem ([7], Lemma 2.1) may be weakened to $f: X \rightarrow E$ with $f(\partial X) \subseteq X$. As a consequence, it is shown that Theorem 2.I, [7], remains true on domains that are not necessarily bounded or quasi-complete.

$$
1 \text {. }
$$

Throughout this paper, let $E$ be a locally convex, Hausdorff topological vector space, $X$ a nonempty subset of $E$, and $U$ a neighborhood base of the origin consisting of absolutely convex subsets of $E$. For each $U \in U$, let $p_{U}$ be the Minkowski's functional of $U$ in

Received 21 April 1976. 
$E$. Note that, since for any finite family $\left\{U_{i}: i=1,2, \ldots, n\right\} \subseteq u$, there is a $V \in U$ with $V \subseteq n\left\{U_{i}: i=1,2, \ldots, n\right\}$, therefore (see [5]),

$$
p_{U_{i}} \leq p_{V} \text { for each } i=1,2, \ldots, n \text {. }
$$

For $x, y \in E$, let

$$
(x, y)=\{z \in E: z=\alpha x+(1-\alpha) y, 0<\alpha<1\},
$$

and $[x, y)=\{x\} \cup(x, y)$. For any subset $A$ of $E$, let $\operatorname{co}(A)$ denote the convex hull of $A, \operatorname{cl}(A)$ the closure of $A$, and $\partial A$ the boundary of $A$ in $E$.

A mapping $f: X \rightarrow E$ is a P-contraction (see [3]) iff for each $p \in P$, there exists a $\alpha_{p}<1$ such that $p(f(x)-f(y)) \leq \alpha_{p} p(x-y)$ for all $x, y \in X$. If this inequality holds with $\alpha_{p} \equiv 1$ for each $p \in P$, then $f$ is called a P-nonexpansive mapping (see [6], [7]). Note that a $P$-contraction or a $P$-nonexpansive mapping is continuous.

The following result is proved in [6] and is used in this paper.

LEMMA 1. Let $X$ be a closed subset of $E$. If $x \in X$ and $y \vDash X$, then there exists $a z \in[x, y) \cap \partial X$; that is $z=(1-\lambda) x+\lambda y \in \partial X$ for some $\lambda \in[0,1)$.

Now let $X$ be a closed subset of $E$ and $f: X \rightarrow E$ be a mapping. An orbit $O(x, f)$ of any $x \in X$ is a sequence $\left\{x_{n}: n \in I, x_{0}=x\right\} \subseteq X$ defined inductively as follows: let $x_{0}=x$ and for each $n \in I$, if $f\left(x_{n}\right) \in X$, set $x_{n+1}=f\left(x_{n}\right)$ and if $f\left(x_{n}\right) \notin X$, then let $x_{n+1}$ be any element of $\left[x_{n}, f\left(x_{n}\right)\right) \cap \partial X \quad$ (such a $x_{n+1}$ exists by Lemma 1$)$.

It follows from the above definition that for each $n \in I$, there is a $\mathbf{r}_{n} \in[0,1]$ such that

$$
x_{n+1}=\gamma_{n} x_{n}+\left(1-\gamma_{n}\right) f\left(x_{n}\right)
$$

Note that if $f\left(x_{n}\right) \notin X$, then $x_{n+1} \in \partial X$. Therefore, if $f(\partial X) \subseteq X$ then $f\left(x_{n}\right) \notin X$ implies $f\left(x_{n+1}\right) \in X$. Also, note that a point may have many 
orbits. However, if $f: X \rightarrow X$, then for any $x \in X$,

$$
O(x, f)=\left\{f^{n}(x): n \in I, f^{0}(x)=x\right\} \text {. }
$$

The following lemma simplifies the proof of the next theorem.

LEMMA 2. Let $X$ be a closed subset of $E$ and let $f: X \rightarrow E$ be a mapping such that $f(\partial X) \subseteq X$. If

(a) $f$ is P-nonexpansive mapping then for any $p \in P$ and oxbit

$$
\begin{aligned}
& o(x, f) \equiv\left\{x_{n}: n \in I, x_{0}=x\right\} \text { of } x \in X, \text { then } \\
& p\left(x_{n}-f\left(x_{n}\right)\right) \leq p(x-f(x)) \text { for each } n \in I \text {, and if }
\end{aligned}
$$

(b) $f$ is a P-contraction,

then for each $p \in P$ and $\varepsilon>0$, there exists $a x \in X$ such that $p(x-f(x))<\varepsilon$.

Proot. Let $O(x, f)=\left\{x_{n}: n \in I, x_{0}=x\right\}$ be an orbit of $x \in X$ and let $p \in P$. Then by (2),

(3) $x_{n+1}-x_{n}=\left(1-\gamma_{n}\right)\left(f\left(x_{n}\right)-x_{n}\right)$ and $x_{n+1}-f\left(x_{n}\right)=\gamma_{n}\left(x_{n}-f\left(x_{n}\right)\right)$.

Since for each $n \in I$,

$$
p\left(x_{n+1}-f\left(x_{n+1}\right)\right) \leq p\left(x_{n+1}-f\left(x_{n}\right)\right)+p\left(f\left(x_{n}\right)-f\left(x_{n+1}\right)\right),
$$

therefore, in either case $(a)$ or (b), it follows by (3) that

(4) $\quad p\left(x_{n+1}-f\left(x_{n+1}\right)\right) \leq p\left(x_{n}-f\left(x_{n}\right)\right) \leq \ldots \leq p\left(x_{0}-f\left(x_{0}\right)\right)=p(x-f(x))$.

This proves $(a)$. To prove $(b)$, it suffices to show that $p\left(x_{n}-f\left(x_{n}\right)\right) \rightarrow 0$. Now, by (4), $\left\{p\left(x_{n}-f\left(x_{n}\right)\right)\right\}$ is a noninereasing sequence of nonnegative reals and hence there is $r \geq 0$ such that $p\left(x_{n}-f\left(x_{n}\right)\right)+r$. We show that $r=0$. Suppose $r>0$. Choose an $\varepsilon>0$ and a $n_{0} \in I$ such that $\alpha_{p}(r+\varepsilon)<r$ and $p\left(x_{n}-f\left(x_{n}\right)\right)<r+\varepsilon$ for all $n \geq n_{0}$. Now choose a $m \in I, m \geq n_{0}$, such that $x_{m+1}=f\left(x_{m}\right)$. (Let $m=n_{0}$ if $f\left(x_{n_{0}}\right) \in X$ and if $f\left(x_{n_{0}}\right) \notin X$, let $m=n_{0}+1$. Note $f\left(x_{n_{0}+1}\right) \in X$, see the remark before Lemma 2.) Then for this $m \in I$,

$$
r \leq p\left(x_{m+1}-f\left(x_{m+1}\right)\right) \leq \alpha_{p} p\left(x_{m}-f\left(x_{m}\right)\right) \leq \alpha_{p}(r+\varepsilon)<r,
$$


which contradicts that $r>0$. Thus $p\left(x_{n}-f\left(x_{n}\right)\right) \rightarrow 0$.

A subset $X$ of $E$ is called starshaped if there exists a $q \in X$ such that for each $x \in X$ and $\gamma \in[0,1], \gamma q+(1-\gamma) x \in X$. The element $q$ is called a star center of $X$.

The following result improves a result of Göhde [4] and also Lemma 2.I in [7].

THEOREM 1. Let $X$ be a closed, starshaped subset of $E$ and let $f: X \rightarrow E$ be a P-nonexpansive mopping such that $f(\partial X) \subseteq X$. Suppose the set $f(X)$ bounded and $f$ satisfies the condition:

(5) there exists a compact subset $L \subseteq X$ such that for each $x \in X$, there is an orbit $O(x, f)$ with $\operatorname{cl}(O(x, f)) \cap L \neq \emptyset$.

Then $\{x \in X: f(x)=x\}$ is a nonempty compact subset of $L$.

Proof. Let $p$ be a fixed element of $P$. First, we show that for each $\varepsilon>0$, there exists an $x \in L$ such that

$$
p(x-f(x))<\varepsilon .
$$

Let $q$ be a star center of $X$. Since, by hypothesis, $f(X)$ is a bounded subset of $E$, therefore there exists an $N \in I$ such that

$$
\frac{1}{N} \sup \{p(q-f(x)): x \in X\}<\frac{\varepsilon}{4}
$$

Define a mapping $g: X \rightarrow E$ by

$$
g(x)=\frac{1}{N} q+\left(1-\frac{1}{N}\right) f(x) .
$$

Then $g$ is a P-contraction $\left(\alpha_{p} \equiv 1-\frac{1}{N}\right.$ for each $\left.p \in P\right)$ and since $f(\partial X) \subseteq X$, it follows by (8) that $g(\partial X) \subseteq X$. Thus, by Lemma $2(b)$, there is a $y \in X$ satisfying $p(y-g(y)) \leq \frac{\varepsilon}{4}$ and hence, by (7) and (8),

$$
p(y-f(y)) \leq p(y-g(y))+p(g(y)-f(y)) \leq \frac{\varepsilon}{4}+\frac{l}{N} p(q-f(y)) \leq \frac{\varepsilon}{2} .
$$

For this $y \in X$, let $O(y, f)=\left\{y_{n}: n \in I, y_{0}=y\right\}$ be an orbit of $y$ such that $\operatorname{cl}(O(y, f)) \cap L \neq \varnothing$. This implies that there is a $y_{n} \in O(y, f)$ and an $x \in L$ such that 


$$
p\left(x-y_{n}\right) \leq \frac{\varepsilon}{4}
$$

Since $f$ if P-nonexpansive, it follows, by Lemma $2(a),(9)$, and (10), that

$$
p(x-f(x)) \leq p\left(x-y_{n}\right)+p\left(y_{n}-f\left(y_{n}\right)\right)+p\left(f\left(y_{n}\right)-f(x)\right) \leq \frac{\varepsilon}{2}+p(y-f(y))<\varepsilon .
$$

This proves (6). It now follows by (6) that for each $n \in I, n \geq 1$, there is an $x_{n} \in L$ such that

$$
p\left(x_{n}-f\left(x_{n}\right)\right) \leq \frac{1}{n}
$$

Since $L$ is compact, there is a subnet $\left\{x_{n}^{\prime}\right\}$ of the net $\left\{x_{n}: n \in I, n \geq 1\right\}$ and an $x_{0} \in L$ such that $\left\{x_{n}^{\prime}\right\} \rightarrow x_{0}$, and it follows, by (11), that

$$
p\left(x_{0}-f\left(x_{0}\right)\right)=0
$$

Thus, for each $p \in P$, there exists an $x_{p} \in L$ such that

$$
p\left(x_{p}-f\left(x_{p}\right)\right)=0 .
$$

Let, for each $p \in P, A_{p}=\{x \in L: p(x-f(x))=0\}$ and $F=\left\{A_{p}: p \in P\right\}$. Then, by (12), $A_{p}$ is a nonempty, closed subset of $L$ for each $p \in P$. Further, since for any finite subset $\left\{p_{i}: i=1,2, \ldots, n\right\} \subseteq P$, there exists a $p \in P$ with $p_{i} \leq p$ for each $i$ (see the first paragraph of Section 1); therefore

$$
A_{p} \subseteq n\left\{A_{p_{i}}: i=1,2, \ldots, n\right\} \text {. }
$$

Since, by (12), $A_{p} \neq \varnothing$, it follows that the family $F$ has a finite intersection property. Consequently $F=\cap\left\{A_{p}: p \in P\right\}$ is a nonempty compact subset of $L$. Clearly $F \subseteq\{x \in X: f(x)=x\}$. Also, if for some $x \in X, f(x)=x$, then $\operatorname{cl}(O(x, f))=\{x\}$ and hence, by (5), $x \in L$, and since $p(x-f(x))=0$ for each $p \in P$, it follows that $x \in F$. Thus $F=\{x \in X: f(x)=x\}$.

REMARK. It may be remarked that Theorem 1 extends Lemma 2.1 in [7], 
where boundedness of $X$ is crucial in the construction of norm \|\|$_{B}$ for the proof. Our proof does not require such a construction of the norm and appears simpler even in this more general case.

2 .

The purpose of this section is to show that the conditions on $X$ being bounded and quasi-complete in Theorem 2.1 in [7] are unnecessary.

The following lemma, whose proof is given in ([7], Lemma 2.2), is stated here for completeness.

LEMMA 3. Let $L$ be a compact subset of $E$. If for some $p \in P$,

$$
d_{p}=\sup \{p(x-y): x, y \in L\}>0 \text {. }
$$

Then there exists $u \in \operatorname{co}(L)$ such that

$$
r=\sup \{p(x-u): x \in L\}<d_{p}
$$

The proof of the following result is similar to the argument in Bakhtin [1].

THEOREM 2. Let $X$ be a nonempty, closed, and convex subset of $E$ and $\Gamma$ a commutative farily of $P$-nonexpansive self mappings of $X$ satisfying the condition:

(14) there exists a $g \in \Gamma$ and a compact set $L \subseteq X$ such that

(i) $g(X)$ is bounded and

(ii) for each $x \in X, \operatorname{cl}\left\{g^{n}(x): n \in I\right\} \cap L \neq \emptyset$.

Then the family $\Gamma$ has a common fixed point in $L$.

Proot. Let

$A=\{S \subseteq X: S$ is nonempty, closed, convex, and $f(S) \subseteq S$ for each $f \in \Gamma\}$.

Then $x \in A$. Define a partial order $\leq$ in $A$ by $S_{1} \leq S_{2}$ iff $S_{2} \subseteq S_{1}$.

We show that each chain in $A$ has an upper bound in $A$. Let

$\left\{S_{\alpha}: \alpha \in \Delta\right\}$ be a chain in $A$. Let $A=\cap\left\{S_{\alpha}: \alpha \in \Delta\right\}$. We show that

$A \neq \emptyset$. For each $\alpha \in \Delta$, set $L_{\alpha}=S_{\alpha} \cap L$. Since $S_{\alpha}$ is closed and

$g\left(S_{\alpha}\right) \subseteq S_{\alpha}$, it follows from (14) (ii) that $L_{\alpha}$ is a nonempty compact 
subset of $S_{\alpha}$ and for any $x \in S_{\alpha}, \operatorname{cl}\left\{g^{n}(s): n \in I\right\} \cap L_{\alpha} \neq \emptyset$. Since $g\left(S_{\alpha}\right)$ is bounded, it follows by Theorem 1 , that for each $\alpha \in \Delta$,

$$
F_{\alpha}=\left\{x \in S_{\alpha}: g(x)=x\right\}
$$

is a nonempty compact subset of $S_{\alpha}$. Since $\left\{F_{\alpha}: \alpha \in \Delta\right\}$ is a chain of compact subsets of $X$, therefore $F=\cap\left\{F_{\alpha}: \alpha \in \Delta\right\} \neq \emptyset$ and $F \subseteq A$. Thus $A \neq \varnothing$. Now it is easy to verify that $A \in A$ and that $A$ is an upper bound of the chain $\left\{S_{\alpha}: \alpha \in \Delta\right\}$. Therefore, by Zorn's Lemma, there exists a maximal element $S_{0} \in A$. Let

$$
F=\left\{x \in S_{0}: g(x)=x\right\} \text {. }
$$

Then, by the similar arguments used above, it follows that $F$ is a nonempty compact subset of $S_{0}$. Further, since for any $f \in \Gamma$ and $x \in F$,

$$
f(x)=f(g(x))=g(f(x)),
$$

it follows that $f(F) \subseteq F$ for each $f \in \Gamma$. Let

$$
B=\left\{C \subseteq S_{0}: C \text { is nonempty, compact, and } f(C) \subseteq C \text { for each } f \in \Gamma\right\} \text {. }
$$

Then $F \in B$. Define the same partial order in $B$ as in $A$. Then it follows by Zorn's Lemma that there is a maximal element $M \in B$. Clearly

$$
M \subseteq S_{0}
$$

Also the maximality of $M$ in $B$ implies that

$$
f(M) \equiv M
$$

for each $f \in \Gamma$, for if $f_{0}(M)=M_{1} \subseteq M$ for some $f_{0} \in \Gamma$, then for each $f \in \Gamma, f\left(M_{1}\right)=f\left(f_{0}\left(M_{1}\right)\right)=f_{0}\left(f\left(M_{1}\right)\right) \subseteq f_{0}(M) \subseteq M_{1}$, contradicting the maximality of $M$ in $B$. We show that $M$ consists of a single element. Suppose not. Then, since $E$ is Hausdorff, there exists a $p \in P$ satisfying

$$
d_{p}=\sup \{p(x-y): x, y \in M\}>0,
$$

and hence by Lemma 3 , there exists a $u \in \operatorname{co}(M)$ such that 


$$
r=\sup \{p(x-u): x \in M\}<d_{p} \text {. }
$$

Since $S_{0}$ is convex, therefore, by (15), $u \in S_{0}$. Let for each $x \in M$,

$$
V(x)=\{z \in E: p(x-z) \leq r\} .
$$

Then $V(x)$ is a closed and convex subset of $E$ and, by (19), $u \in V(x)$ for each $x \in M$. Set

$$
V=n\{V(x): x \in M\} \text { and } S=S_{0} \cap V \text {. }
$$

Clearly $S$ is a closed and convex subset of $X$ and $u \in S$. We shall show that $f(S) \subseteq S$ for each $f \in \Gamma$. Since $f\left(s_{0}\right) \subseteq S_{0}$ for each $f \in \Gamma$, it suffices to show that $f(V) \subseteq V$ for each $f \in \Gamma$. Let $z \in V$ and $f \in \Gamma$. Then, by (20),

$$
p(x-z) \leq r
$$

for each $x \in M$. Now by (17), for each $x \in M$, there is a $y=y(x) \in M$ such that $f(y)=x$ and hence, by (22),

$$
p(f(z)-x)=p(f(z)-f(y)) \leq p(z-y) \leq r,
$$

for each $x \in M$. The last inequality implies that $f(z) \in V(x)$ for each $x \in M$; that is $f(V) \subseteq V$ and consequently $f(S) \subseteq S$ for each $f \in \Gamma$. Thus $S \in A$. However, by $(21), S_{0} \leq S$ and since $S_{0}$ is maximal in A,

$$
s_{0}=S
$$

Now $p$ being continuous and $M$ compact, there are elements $x, y \in M$ such that $p(x-y)=d_{p}$. Since $r<d_{p}$, the last equality implies that $y \notin V(x)$ and hence $y \notin S$. However, since $M \subseteq S_{0}, y \in S_{0}$. This contradicts (23). Thus $M=\{x\}$ for some $x \in X$. This implies that $f(x)=x$ for each $f \in \Gamma$.

\section{References}

[1] A. Bakhtin, "Existence of common fixed points for comutative sets of nonlinear operators", Functional Anal. App 2. 4 (1970), 76-77.

[2] L.P. Belluce and W.A. Kirk, "Fixed-point theorems for families of contraction mappings", Pacific J. Math. 18 (1966), 213-217. 
[3] G.L. Cain, Jr. and M.Z. Nashed, "Fixed points and stability for a sum of two operators in locally convex spaces", Pacific J. Math. 39 (1971), 581-592.

[4] Dietrich Göhde, "Über Fixpunkte bei stetigen Selbstabbildugen mit kompakten Iterierten", Math. Nachr. 28 (1964/65), 45-55.

[5] A.P. Robertson and Wendy Robertson, Topological vector spaces (Cambridge Tracts in Mathematics and Mathematical Physics, 53. Cambridge University press, Cambridge, 1964; reprinted, 1966).

[6] C.H. Su, V.M. Sehgal, "Some fixed point theorems for nonexpansive mappings in locally convex spaces", Bolz. Un. Mat. Ital. (4) 10 (1974), 598-601.

[7] E. Tarafdar, "Some fixed-point theorems on Iocally convex linear topological spaces", Bulz. Austral. Math. Soc. 13 (1975), 241-245.

[8] W.W. Taylor, "Fixed-point theorems for nonexpansive mappings in linear topological spaces", J. Math. Anal. Appl. 40 (1972), 164-173.

Department of Mathematics,

University of Wyoming,

Laramie,

Wyoming,

USf. 